\title{
Roles of sex-determining region Y-box 2 in cell pluripotency and tumor-related signaling pathways (Review)
}

\author{
JINGJIE WANG, HUIJUAN ZENG, HANJUN LI, JUANJUAN ZHANG and SHAOHUA WANG \\ Department of General Surgery, Jinling Hospital, Medical School of Nanjing University, \\ Nanjing, Jiangsu 210002, P.R. China
}

Received March 27, 2015; Accepted August 14, 2015

DOI: $10.3892 / \operatorname{mco} .2015 .639$

\begin{abstract}
The sex-determining region Y-box 2 (SOX2) gene, a member of the Sry-like high-mobility group box (SOX) gene family, encodes the transcription factor Sox2, which significantly contributes to the regulation of cell pluripotency. Sox 2 is closely associated with early embryonic development, neural differentiation and other biological processes. An inreasing number of recent studies suggest that Sox 2 exerts a positive effect on malignant tumors. According to these results, Sox 2 is expected to become a novel target for cancer therapy by unveiling the mechanism through which it affects the biological behavior of tumors. Therefore, it is crucial to elucidate the detailed association of Sox 2 with malignant tumors. The aim of this study was to review the role of Sox 2 in pluripotency maintenance, early embryonic development and neural differentiation, as well as investigate the detailed mechanism through which Sox 2 regulates cancer stem cells and tumorigenesis.
\end{abstract}

\section{Contents}

1. Introduction

2. Association between Sox 2 and cancer stem cells (CSCs)

3. Correlation between Sox 2 and malignant tumors in clinical statistics

4. Role of Sox 2 in tumor-related signaling pathways

5. Conclusion and perspectives

Correspondence to: Professor Shaohua Wang, Department of General Surgery, Jinling Hospital, Medical School of Nanjing University, 305 East Zhongshan Road, Nanjing, Jiangsu 210002, P.R. China

E-mail: wanglaifu2@126.com

Abbreviations: Sox2, sex-determining region Y-box 2; SOX, Sry-like high-mobility group box; CSCs, cancer stem cells; PI3K, phosphatidylinositol 3-kinase

Key words: sex-determining region Y-box 2, pluripotency, cancer stem cells, malignant tumor, signaling pathway

\section{Introduction}

Characteristics of sex-determining region Y-box 2 (Sox2). The transcription factor Sox 2 belongs to the high-mobility group superfamily and is encoded by the SOX2 gene, a member of the SOX gene family, the members of which are conserved across species and involved in a number of developmental processes (1). Since the discovery of the first SOX gene, $\sim 20$ genes of the SOX gene family have been identified over the last 20 years in mammals and classified into groups according to protein specificity (2). The SOX2 gene was discovered and characterized in humans in 1994; it belongs to the SOXB1 group, which also includes SOX1 and SOX3. The SOXB1 group locates on chromosome 3q26.3-q27 and encodes a protein consisting of 317 amino acids $(3,4)$. Although there are $>80 \%$ sequence similarities across the SOXB1 family, SOX2 plays a distinct role in complex biological progress in a context-dependent manner, and is an indispensable factor for embryonic development.

Function of Sox2. Sox 2 is well known as one of the pluripotency factors, also including Oct4, Nanog and Klf4. Sox2 contributes significantly to the regulation of cell pluripotency and is closely associated with early embryonic development, neural differentiation and sexual differentiation.

Sox2 is crucial for the maintenance of pluripotency in cells. Human pluripotent stem cells (hPSCs) include human embryonic stem cells (hESCs) and induced pluripotent stem cells (iPSCs) (5). These cells possess two important properties, namely limitless self-renewal in culture and the ability to differentiate into different types of blastoderm in the early embryo (6). These unique properties make hPSCs a research focus in the field of personalized medicine, drug screening or cell therapy $(7,8)$ and the study of early human development, serving as a cell model to elucidate the molecular mechanisms regulating embryonic cell proliferation and differentiation (7). hPSCs are regulated by several stem genes, including SOX2. It has been reported that iPSCs were first produced from fibroblasts through regulation of Sox2. Liu et al (8) reported that, in human iPSCs with human amniotic epithelial cells as feeder cells, overexpression of Sox 2 maintained cell pluripotency via inhibition of endogenous microRNA-145 expression. Xu et al (9) also found that microRNA-145 regulates Sox2 and 
represses pluripotency in hESCs. These results indicate that Sox 2 is indispensable in the maintenance of the pluripotency of hPSCs.

Sox 2 plays an important role in embryogenesis. Sox 2 is a factor indispensable to embryonic development. It was previously indicated that the main function of Sox 2 is to help maintain the pluripotency of ESCs and repress trophectoderm and epiblast genes in later stages of development (10). During mouse embryogenesis, a zygote multiplies via mitosis, resulting in the formation of the multicellular morula (16-32 cells), which further develops to form the blastocyst ( $>32$ cells). The blastoderm in the blastocyst is divided into the inner cell mass (ICM) and the trophectoderm. Cells in the ICM have the ability to differentiate into any type of cell found in the human body. Thus, ICM cells are classified as pluripotent. Sox 2 was first identified in the morula, specifically in the ICM of the blastocyst and epiblast (11). Lack of Sox2 expression significantly affects the formation of the trophectoderm (11), indicating that Sox 2 plays an important role in the formation of the embryo and the maintainance of cell pluripotence.

Sox 2 plays an essential role in neural differentiation. Sox 2 also appears to play an important role in the differentiation of the nervous system (10). Sox 2 is expressed not only in ESCs, but also in several other types of stem cells, including neural stem cells, and it regulates the progress of primary neurogenesis, neural development and neural differentiation. Mizuseki et al (12) indicated in 1998 that Sox 2 alone is not sufficient to induce neural tissue differentiation in ectodermal explants excised from blastulas. In 2009, Archer et al (13) demonstrated that there is an interaction among Sox1, Sox2, Sox 3 and Oct 4 during primary neurogenesis. Only Sox1 and Oct91 induce the formation of neurons; Sox2, however, is more effective compared with Sox 1 in maintaining cells in a progenitor state. While the SoxB1s have overlapping functions, they are not strictly redundant, as they induce different sets of genes and are likely to partner with different proteins to maintain progenitor identity. Therefore, Sox 2 is an important factor, although not the only factor, for primary neurogenesis, and it acts synergistically with other members of the SoxB1 family.

\section{Association between Sox 2 and cancer stem cells (CSCs)}

CSCs, also referred to as tumor-initiating cells or cancer stem-like cells, have been experimentally defined by their ability to initiate tumor formation upon implantation in immunocompromised mice and are considered to be an important factor in tumorigenesis, invasion and metastasis. Sox 2 acts in CSCs via a number of signaling pathways. In 2012 , Basu-Roy et al (14) reported that Sox 2 is highly expressed in human and murine osteosarcoma cell lines, as well as in tumor samples, and is essential for the self-renewal of osteoblast progenitor cells. However, Sox 2 antagonizes the Wnt pathway, which is a pro-differentiation pathway that may, in turn, reduce Sox 2 expression. Rybak et al (15) reported in 2013 that the activation of epidermal growth factor receptor (EGFR) signaling pathways in prostate cancer stem cells (PCSCs) contributes to a significant upregulation of SOX2 at both the mRNA and the protein levels, promoting the self-renewal of DU145 PCSCs. In addition, other studies indicated that the SOX2 expression status of CSCs is the basis of different pathological types of tumors $(16,17)$. Therefore, Sox 2 is an important factor in tumorigenesis via CSC amplification.

\section{Correlation between Sox 2 and malignant tumors in clinical statistics}

An increasing number of studies suggest that Sox 2 exerts a positive effect on pluripotency regulation, tumorigenesis, tumor invasion and metastasis in breast cancer (18), melanoma (19), laryngeal carcinoma (20), hepatocellular carcinoma (HCC) (21), ovarian cancer (22) and non-small-cell lung cancer (23).

Huang et al (18) evaluated 57 ductal carcinomas in situ, 552 invasive breast carcinomas and 107 corresponding metastatic lymph nodes and found that Sox 2 expression was closely associated with clinicopathological parameters, including high histological grade, large tumor size, molecular subtypes with adverse outcome, negative hormone receptor status, high proliferation index and neuroendocrine marker expression.

Sun et al (21) evaluated 75 HCCs and discovered that high levels of Sox 2 expression were correlated with metastasis and low survival rate in HCC. They also investigated the molecular role of Sox 2 in human HCC cell lines with stepwise metastatic potential (MHCC97L, MHCC97H and MHCCLM3) and found that Sox 2 activates epithelial-to-mesenchymal transition (EMT) in HCC cells (21).

Zhang et al (24) investigated the clinical role of Sox2 expression in ovarian carcinoma. Immunohistochemical staining of 540 human ovarian carcinoma samples for Sox 2 was performed using tissue microarray and the results suggested that Sox 2 expression was associated with high-grade carcinomas, particularly high-grade serous carcinoma, International Federation of Gynecology and Obstetrics stage and malignant mixed Müllerian tumors $(\mathrm{P}=0.048)$.

Several other malignant tumor clinical analyses have been performed in recent years on lung cancer, melanoma and brain tumors, providing evidence that Sox 2 is closely associated with tumor invasion and metastasis and predicts poor prognosis in malignant tumor patients. However, the underlying mechanism remains to be elucidated.

\section{Role of Sox 2 in tumor-related signaling pathways}

Sox 2 regulates tumor growth, invasion and metastasis via a plurality of signaling pathways, including the phosphatidylinositol 3-kinase (PI3K)/protein kinase B (Akt), Wnt/ $\beta$-catenin, Notch, bone morphogenetic protein (BMP), estrogen receptor $\alpha(E R \alpha)$ and mitogen-activated protein kinase kinase kinase kinase 4 (MAP4K4)-survivin signaling pathway. These signaling pathways form a complex regulatory network, indicating that Sox 2 plays a decisive role in tumor bioinformatics (Table I).

PI3K/Akt signaling pathway. The PI3K/Akt and related signaling pathways are among the most well-known tumor-related signaling pathways; they are important in internalizing the effects of external growth factors and 
Table I. Signaling pathways associated with Sox2 in different malignant tumors.

\begin{tabular}{|c|c|c|}
\hline Signaling pathways & Related malignant tumors & References \\
\hline \multirow[t]{4}{*}{ PI3k/Akt } & Prostate cancer & $(26),(27)$ \\
\hline & Hepatocellular carcinoma & (28) \\
\hline & Breast cancer & $(29)$ \\
\hline & Esophageal squamous cell carcinoma & $(30)$ \\
\hline \multirow[t]{4}{*}{ Wnt/ $\beta$-cantenin } & Prostate cancer & $(32)$ \\
\hline & Glioblastoma & (33) \\
\hline & Breast cancer & $(32)$ \\
\hline & Laryngeal cancer & $(34)$ \\
\hline \multirow[t]{2}{*}{ Notch } & Nasopharyngeal carcinoma & $(38)$ \\
\hline & Lung cancer & $(17),(39)$ \\
\hline \multirow[t]{2}{*}{ BMP } & Colorectal cancer & $(40)$ \\
\hline & Lung squamous cell carcinoma & $(41)$ \\
\hline $\mathrm{ER} \alpha$ & Breast cancer & $(42)$ \\
\hline MAP4K4-survivin & Lung cancer & $(43)$ \\
\hline
\end{tabular}

Sox2, sex-determining region Y-box 2; PI3k, phosphatidylinositol 3-kinase; BMP, bone morphogenetic protein; ER $\alpha$, estrogen receptor $\alpha$; MAP4K4, mitogen-activated protein kinase kinase kinase kinase 4.

membrane tyrosine kinases. The activation of membrane kinases, including EGFR, by external growth factors initiates receptor dimerization and subsequent events to activate these intracellular pathways. PI3K participates in the regulation of proliferation, differentiation, apoptosis, glucose transport and other cellular functions. The most extensively investigated member of the PI3K family is type I PI3K, which may be activated by cell surface receptors. In mammalian cells, type I PI3K includes two subtypes, namely IA and IB. Recent publications indicated that signaling pathways including IA and its downstream molecules, such as Akt, are closely associated with the occurrence and development of malignant tumors. This pathway regulates tumor cell proliferation and survival; its abnormal activity not only causes cell malignant transformation, but is also associated with tumor cell migration, adhesion, angiogenesis and extracellular matrix degradation. Activated Akt regulates the expression of downstream molecules, including mammalian target of rapamycin (mTOR), extracellular signal-regulated kinase, Forkhead, guanosine diphosphate, insulin receptor substrate, glycogen synthase kinase 3 (GSK3), mitogen-activated protein kinase, nuclear factor-B, protein kinase $\mathrm{C}$ and signal transducer and activator of transcription, and then regulates cell proliferation, differentiation, apoptosis and migration. Sox 2 was recently identified as a novel regulator of the PI3K/Akt pathway. Yu et al (25) reported in 2012 that Sox 2 promotes the expression of CD49f, which regulates the ability of hMSCs to form spheres via activating the PI3K/Akt signaling pathway.

The role of the PI3K/Akt signaling pathway in malignant tumors is attracting increasing attention in the field of clinical and preclinical medicine. Morgan et al (26) reported in 2009 the prominent role of the PI3K/Akt/mTOR pathway in prostate cancer. Molecular changes in the PI3K/Akt/mTOR signaling pathway have been demonstrated to distinguish benign from malignant prostatic epithelium and are associated with increasing tumor stage, grade and risk of biochemical recurrence. In addition, the PI3K/Akt/mTOR signaling pathway is associated with drug resistance in prostate cancer. Li et al (27) investigated the PC-3 prostate cancer cell line in 2013 and found that overexpression of Sox 2 enhanced the resistance of PC-3 cells to paclitaxel through promoting cell proliferation and exhibiting an anti-apoptotic effect via activation of the PI3K/Akt pathway. Another study on HCC demonstrated that cyclin G1 expands liver tumor-initiating cells through Sox 2 induction via Akt/mTOR signaling (28). Similar results were reported in breast cancer (29) and esophageal squamous cell carcinoma (SCC) (30), suggesting that Sox 2 is correlated with CSCs via the PI3K/Akt pathway, although the detailed mechanism remains to be elucidated.

Wnt/ $\beta$-catenin signaling pathway. The Wnt signaling pathway is among the most well-known tumor-related signaling pathways and it is a complex protein interaction network, most commonly involved in embryonic development and tumorigenesis. Three pathways, including the Wnt/ $\beta$-catenin, planar cell polarity and $\mathrm{Wnt} / \mathrm{Ca}^{2+}$ pathways, act synergistically but independently in physiological processes. Among these, the Wnt/ $\beta$-catenin pathway is the one most closely associated with tumorigenesis. Activation of this pathway stabilizes $\beta$-catenin and, therefore, contributes to accumulation of this protein, which then enters the nucleus to activate the expression of target genes. In the absence of secreted glycolipoprotein Wnt, cytoplasmic $\beta$-catenin protein is constantly degraded by the action of the Axin complex, which is composed of the scaffolding protein Axin, the tumor suppressor adenomatous polyposis coli gene product, casein kinase 1 and GSK3 (31). Sox 2 is a negative regulator of the Wnt pathway via binding to $\beta$-catenin and inducing the Wnt inhibitor Dickkopf-related protein (Dkk) 1. Li et al (32) reported that Sox2 promotes metastasis of breast and prostate cancer cells by promoting 
EMT through WNT/ $\beta$-catenin. In addition, Sox 2 affects the protein expression levels of Dkk3, dishevelled segment polarity protein (DVL) 1 and DVL3, which are regulators or downstream molecules of Wnt signaling (32). A previous study by Berezovsky et al (33) on glioblastoma multiforme (GBM) indicates that Sox 2 reprograms differentiated cells into pluripotent cells and regulates the expression of key genes and Wnt pathways involved in GBM in cancer stem-like and differentiated cells. Another study by Yang et al (34) on HEp-2 laryngeal cancer cells revealed that the overexpression of Sox 2 promotes cancer cell migration, invasion and EMT through the Wnt/ $\beta$-catenin pathway. However, the detailed association between the Sox 2 and Wnt pathways has not been elucidated.

Notch signaling pathway. The NOTCH gene was firstly discovered in Drosophila, in which partial loss of function of this gene caused a notch of the wing edge. The Notch signaling pathway is a highly conserved transduction pathway in the evolution and function of cell proliferation, differentiation and apoptosis regulation, and is involved in almost all tissues and organs. Notch is a transmembrane receptor, widely present in almost all known animal cells. The Notch pathway mediates cell-cell signal transfer and the corresponding signaling cascade reaction. The Notch signaling pathway is composed of Notch, Notch ligand (DSL protein) and CSL (a DNA-binding protein). Notch and its ligands are all single transmembrane proteins. Notch is cut by proteasome when the ligand binds to Notch of the adjacent cell, releasing intracellular Notch (ICN), which carries the nuclear localization signal in the cytoplasmic region. ICN then enters the nucleus and binds to CLS to regulate target gene expression. To date, the function of the Notch signaling pathway in the regulation of cell proliferation, differentiation and apoptosis has been extensively investigated, particularly in the inner ear (35-37). However, the number of studies on the interaction between Sox 2 and the Notch pathway in tumor invasion and metastasis is limited. Zhang et al (38) investigated Notch1 signaling in nasopharyngeal carcinoma (NPC) and found that the Notch1 signaling-activated form was predominantly found in Sox2-negative cells. This finding indicated that the overexpression of Sox 2 inhibits Notch1 signaling and contributes to the pathological self-renewal characteristics of CSCs in human NPC. By contrast, Chen et al (17) found that NOTCH3, c-Myc and WNT7B were significantly reduced after silencing SOX2 in A549 human lung cancer cells, suggesting that Notch signaling is the downstream pathway of Sox2. Another research on lung cancer revealed that the overexpression of Sox 2 was able to inhibit Notch signaling in CC10+ cells, subsequently inhibiting K-Ras-induced tumor formation; inhibition of Notch signaling strongly inhibits adenocarcinoma formation, but promotes squamous hyperplasia in the alveoli (39). The abovementioned research results demonstrated that Sox 2 may regulate the activity of the Notch signaling pathway and carcinogenesis. Of note, Sox 2 may not only activate but also inhibit the Notch pathway, indicating that Sox 2 exerts a two-way regulatory effect on Notch. The detailed mechanism, however, remains to be elucidated.

Other signaling pathways. The PI3K/Akt, Wnt/ $\beta$-catenin and Notch signaling pathways are the most well-known pathways involved in tumorigenesis. In addition, there are more signaling pathways correlated with Sox 2 in regulating carcinogenesis. Fang et al (40) used ChIP-seq and functional analysis in SW620 colorectal cancer cells and found that Sox2 is associated with the BMP signaling pathway and a number of receptor-mediated signaling pathways, such as insulin-like growth factor 1 receptor and inositol 1,4,5-triphosphate receptor, type 2 . A study conducted in 2014 by Fang et al (41) suggested that BMP4 is a downstream target of Sox 2 in lung SCC. Sox 2 is a negative regulator of BMP4 and overexpression of Sox 2 may reduce the expression of BMP, promoting the occurrence and development of SCC (41). In addition, in breast cancer, Sox 2 was found to be a new target of miRNA140, regulating breast tumor-initiating cells via the ER $\alpha$ signaling pathway (42). Chen et al (43) found that Sox2 regulates apoptosis through the MAP4K4-survivin signaling pathway in human lung cancer cells. In conclusion, Sox 2 plays different roles in the regulation of tumor development, invasion and metastasis and drug resistance. The heterogeneity of research conclusions indicates the complexity of the network associating Sox 2 with tumor-related signaling pathways. Therefore, the specific role of Sox 2 in tumor-associated signaling pathways requires further investigation.

\section{Conclusion and perspectives}

Sox 2 significantly contributes to the regulation of cell pluripotency and is closely associated with early embryonic development, particularly neural differentiation. Due to the similarities of the characteristics and functions between Sox 2 and CSCs, the correlation between Sox 2 and malignant tumors has been attracting increasing attention. An increasing number of studies suggest that Sox 2 exerts a positive effect on pluripotency regulation, tumorigenesis, tumor invasion and metastasis in several types of malignant tumors. Sox 2 regulates tumor growth, invasion and metastasis via a plurality of signaling pathways. These signaling pathways form a complex regulatory network, indicating that Sox 2 plays a decisive role in tumor bioinformatics. Therefore, the roles of Sox 2 in tumors require further elucidation. Sox 2 and its upstream/downstream molecules in the transduction pathway network and the proteins interacting with Sox 2 are expected to be novel targets for cancer therapy; the elucidation of the mechanism underlying the effect of Sox 2 on the biological behavior of tumors is expected to lead to significant advances in cancer treatment.

\section{Acknowledgements}

This study is funded by the National Key Basic Research Program of China (973 Program) grant no. 2014CB744504.

\section{References}

1. Dong C, Wilhelm D and Koopman P: Sox genes and cancer. Cytogenet Genome Res 105: 442-447, 2004.

2. Castillo SD and Sanchez-Cespedes M: The SOX family of genes in cancer development: Biological relevance and opportunities for therapy. Expert Opin Ther Targets 16: 903-919, 2012.

3. Stevanovic M, Zuffardi O, Collignon J, Lovell-Badge R and Goodfellow P: The cDNA sequence and chromosomal location of the human SOX2 gene. Mamm Genome 5: 640-642, 1994.

4. Weina K and Utikal J: SOX2 and cancer: Current research and its implications in the clinic. Clin Transl Med 3: 19, 2014. 
5. Thomson JA, Itskovitz-Eldor J, Shapiro SS, Waknitz MA Swiergiel JJ, Marshall VS and Jones JM: Embryonic stem cell lines derived from human blastocysts. Science 282: 1145-1147, 1998.

6. Masui S, Nakatake Y, Toyooka Y, Shimosato D, Yagi R Takahashi K, Okochi H, Okuda A, Matoba R, Sharov AA, et al: Pluripotency governed by Sox 2 via regulation of Oct3/4 expression in mouse embryonic stem cells. Nat Cell Biol 9: 625-635, 2007

7. Zhang S and Cui W: Sox2, a key factor in the regulation of pluripotency and neural differentiation. World J Stem Cells 6: 305-311, 2014

8. Liu T, Cheng W, Huang Y, Huang Q, Jiang L and Guo L: Human amniotic epithelial cell feeder layers maintain human iPS cell pluripotency via inhibited endogenous microRNA-145 and increased Sox2 expression. Exp Cell Res 318: 424-434, 2012.

9. Xu N, Papagiannakopoulos T, Pan G, Thomson JA and Kosik KS: MicroRNA-145 regulates OCT4, SOX2, and KLF4 and represses pluripotency in human embryonic stem cells. Cell 137: 647-658, 2009.

10. Chew JL, Loh YH, Zhang W, Chen X, Tam WL, Yeap LS, Li P, Ang YS, Lim B, Robson P, et al: Reciprocal transcriptional regulation of Pou5f1 and Sox 2 via the Oct4/Sox 2 complex in embryonic stem cells. Mol Cell Biol 25: 6031-6046, 2005

11. Avilion AA, Nicolis SK, Pevny LH, Perez L, Vivian N and Lovell-Badge R: Multipotent cell lineages in early mouse development depend on SOX2 function. Genes Dev 17: 126-140, 2003

12. Mizuseki K, Kishi M, Matsui M, Nakanishi S and Sasai Y: Xenopus Zic-related-1 and Sox-2, two factors induced by chordin, have distinct activities in the initiation of neural induction. Development 125: 579-587, 1998.

13. Archer TC, Jin J and Casey ES: Interaction of Sox1, Sox2, Sox3 and Oct4 during primary neurogenesis. Dev Biol 350: 429-440, 2011.

14. Basu-Roy U, Seo E, Ramanathapuram L, Rapp TB, Perry JA Orkin SH, Mansukhani A and Basilico C: Sox2 maintains self renewal of tumor-initiating cells in osteosarcomas. Oncogene 31 : 2270-2282, 2012.

15. Rybak AP and Tang D: SOX2 plays a critical role in EGFR-mediated self-renewal of human prostate cancer stem-like cells. Cell Signal 25: 2734-2742, 2013.

16. Boumahdi S, Driessens G, Lapouge G, Rorive S, Nassar D Le Mercier M, Delatte B, Caauwe A, Lenglez S, Nkusi E, et al: SOX2 controls tumour initiation and cancer stem-cell functions in squamous-cell carcinoma. Nature 511: 246-250, 2014.

17. Chen S, Xu Y, Chen Y, Li X, Mou W, Wang L, Liu Y, Reisfeld RA, Xiang R, Lv D, et al: SOX2 gene regulates the transcriptional network of oncogenes and affects tumorigenesis of human lung cancer cells. PLoS One 7: e36326, 2012

18. Huang YH, Luo MH, Ni YB, Tsang JY, Chan SK, Lui PC, Yu AM, Tan PH and Tse GM: Increased SOX2 expression in less differentiated breast carcinomas and their lymph node metastases. Histopathology 64: 494-503, 2014.

19. Girouard SD, Laga AC, Mihm MC, Scolyer RA, Thompson JF Zhan Q, Widlund HR, Lee CW and Murphy GF: SOX2 contributes to melanoma cell invasion. Lab Invest 92: 362-370, 2012.

20. Yang N, Hui L, Wang Y, Yang H and Jiang X: SOX2 promotes the migration and invasion of laryngeal cancer cells by induction of MMP-2 via the PI3K/Akt/mTOR pathway. Oncol Rep 31: 2651-2659, 2014

21. Sun C, Sun L, Li Y, Kang X, Zhang S and Liu Y: Sox2 expression predicts poor survival of hepatocellular carcinoma patients and it promotes liver cancer cell invasion by activating Slug. Med Oncol 30: 503, 2013

22. Lou X1, Han X, Jin C, Tian W, Yu W, Ding D, Cheng L, Huang B, Jiang $\mathrm{H}$ and Lin B: SOX2 targets fibronectin 1 to promote cel migration and invasion in ovarian cancer: New molecular leads for therapeutic intervention. OMICS 17: 510-518, 2013.

23. Xiang R, Liao D, Cheng T, Zhou H, Shi Q, Chuang TS, Markowitz D, Reisfeld RA and Luo Y: Downregulation of transcription factor SOX2 in cancer stem cells suppresses growth and metastasis of lung cancer. Br J Cancer 104: 1410-1417, 2011.

24. Zhang J, Chang DY, Mercado-Uribe I and Liu J: Sex-determining region Y-box 2 expression predicts poor prognosis in human ovarian carcinoma. Hum Pathol 43: 1405-1412, 2012.
25. Yu KR, Yang SR, Jung JW, Kim H, Ko K, Han DW, Park SB, Choi SW, Kang SK, Schöler H, et al: CD49f enhances multipotency and maintains stemness through the direct regulation of OCT4 and SOX2. Stem Cells 30: 876-887, 2012.

26. Morgan TM, Koreckij TD and Corey E: Targeted therapy for advanced prostate cancer: Inhibition of the PI3K/Akt/mTOR pathway. Curr Cancer Drug Targets 9: 237-249, 2009.

27. Li D, Zhao LN, Zheng XL, Lin P, Lin F, Li Y, Zou HF, Cui RJ, Chen $\mathrm{H}$ and Yu XG: Sox 2 is involved in paclitaxel resistance of the prostate cancer cell line PC-3 via the PI3K/Akt pathway. Mol Med Rep 10: 3169-3176, 2014.

28. Wen W, Han T, Chen C, Huang L, Sun W, Wang X, Chen SZ, Xiang DM, Tang L, Cao D, et al: Cyclin G1 expands liver tumor-initiating cells by Sox 2 induction via Akt/mTOR signaling. Mol Cancer Ther 12: 1796-1804, 2013.

29. Corominas-Faja B, Cufí S, Oliveras-Ferraros C, Cuyàs E, López-Bonet E, Lupu R, Alarcón T, Vellon L, Iglesias JM, Leis $\mathrm{O}$, et al: Nuclear reprogramming of luminal-like breast cancer cells generates Sox2-overexpressing cancer stem-like cellular states harboring transcriptional activation of the mTOR pathway. Cell Cycle 12: 3109-3124, 2013.

30. Gen Y, Yasui K, Nishikawa T and Yoshikawa T: SOX2 promotes tumor growth of esophageal squamous cell carcinoma through the AKT/mammalian target of rapamycin complex 1 signaling pathway. Cancer Sci 104: 810-816, 2013

31. Al-Dhfyan A: Embryonic signature in breast cancers; Pluripotency roots of cancer stem cells. Saudi pharmaceutical journal : Saudi Pharm J 21: 229-232, 2013.

32. Li X, Xu Y, Chen Y, Chen S, Jia X, Sun T, Liu Y, Li X, Xiang R and Li N: SOX2 promotes tumor metastasis by stimulating epithelial-to-mesenchymal transition via regulation of WNT/ $\beta$-catenin signal network. Cancer Lett 336: 379-389, 2013.

33. Berezovsky AD, Poisson LM, Cherba D, Webb CP, Transou AD Lemke NW, Hong X, Hasselbach LA, Irtenkauf SM, Mikkelsen T and Carvalho AC: Sox 2 promotes malignancy in glioblastoma by regulating plasticity and astrocytic differentiation. Neoplasia 16: 193-206, 206 e19-25, 2014.

34. Yang N, Hui L, Wang Y, Yang H and Jiang X: Overexpression of SOX 2 promotes migration, invasion, and epithelial-mesenchymal transition through the Wnt/ $\beta$-catenin pathway in laryngeal cancer Hep-2 cells. Tumour Biol 35: 7965-7973, 2014

35. Jeon SJ, Fujioka M, Kim SC and Edge AS: Notch signaling alters sensory or neuronal cell fate specification of inner ear stem cells. J Neurosci 31: 8351-8358, 2011

36. Neves J, Abelló G, Petrovic J and Giraldez F: Patterning and cell fate in the inner ear: A case for Notch in the chicken embryo. Dev Growth Differ 55: 96-112, 2013.

37. Pan W, Jin Y, Chen J, Rottier RJ, Steel KP and Kiernan AE: Ectopic expression of activated notch or SOX2 reveals similar and unique roles in the development of the sensory cell progenitors in the mammalian inner ear. J Neurosci 33: 16146-16157, 2013.

38. Zhang Y1, Peng J, Zhang H, Zhu Y, Wan L, Chen J, Chen X, Lin R, Li H, Mao X, et al: Notch1 signaling is activated in cells expressing embryonic stem cell proteins in human primary nasopharyngeal carcinoma. J Otolaryngol Head Neck Surg 39: $157-166,2010$

39. Xu X, Huang L, Futtner C, Schwab B, Rampersad RR, Lu Y, Sporn TA, Hogan BL and Onaitis MW: The cell of origin and subtype of K-Ras-induced lung tumors are modified by Notch and Sox2. Genes Dev 28: 1929-1939, 2014.

40. Fang X1, Yu W, Li L, Shao J, Zhao N, Chen Q, Ye Z, Lin SC, Zheng $S$ and Lin B: ChIP-seq and functional analysis of the SOX2 gene in colorectal cancers. OMICS 14: 369-384, 2010.

41. Fang WT1, Fan CC, Li SM, Jang TH, Lin HP, Shih NY, Chen CH, Wang TY, Huang SF, Lee AY, et al: Downregulation of a putative tumor suppressor BMP4 by SOX2 promotes growth of lung squamous cell carcinoma. Int J Cancer 135: 809-819, 2014.

42. Zhang Y, Eades G, Yao Y, Li Q and Zhou Q: Estrogen receptor $\alpha$ signaling regulates breast tumor-initiating cells by down-regulating miR-140 which targets the transcription factor SOX2. J Biol Chem 287: 41514-41522, 2012.

43. Chen S, Li X, Lu D, Xu Y, Mou W, Wang L, Chen Y, Liu Y, Li X, Li LY, et al: SOX2 regulates apoptosis through MAP4K4-survivin signaling pathway in human lung cancer cells. Carcinogenesis 35 : 613-623, 2014. 